\title{
COSTO-EFECTIVIDAD DEL TRATAMIENTO QUIRÚRGICO VS CORTICOIDES, LOCALES EN EL SÍNDROME DEL TÚNEL DEL CARPO
}

\section{COST-EFFECTIVENESS OF SURGICAL TREATMENT VS LOCAL STEROIDS IN CARPAL TUNNEL SYNDROME}

\author{
Francisco Palencia-Sánchez ${ }^{1 *}$, Oscar García-Vega ${ }^{2}$, Martha Riaño-Casallas $^{3}$
}

\begin{abstract}
${ }^{1}$ Médico M.Sc. Epidemiología, $\mathrm{PhD}(\mathrm{c})$ en Salud Pública, Investigador en el Doctorado Inter facultades en Salud Pública. Universidad Nacional de Colombia, Carrera 30 No. 45-03, Bogotá, D.C., Facultad de Medicina *autor para correspondencia, e-mail: fpalencias@unal.edu.co; ${ }^{2}$ Médico, M.Sc. Farmacología, Ph.D. en Farmacología, Docente en Facultad de Medicina. Universidad Nacional de Colombia, e-mail: oagarciav@unal.edu.co; ${ }^{3}$ Administradora de empresas, M.Sc. Seguridad y Salud en el Trabajo, PhD en Ciencias Económicas, Docente Facultad de Ciencias Económicas. Universidad Nacional de Colombia, e-mail:mirianoc@unal.edu.co
\end{abstract}

Rev. U.D.C.A Act. \& Div. Cient. 21(1): 5-14, Enero-Junio, 2018

https://doi.org/10.31910/rudca.v21.n1.2018.657

\section{RESUMEN}

El síndrome del túnel del carpo (STC) es la neuropatía por atrapamiento más frecuente $y$, a su vez, la que más afecta a la población trabajadora, cuyo tratamiento implica una carga económica para el sistema de salud. El objetivo de esta investigación fue evaluar el costo-efectividad del tratamiento quirúrgico convencional y la infiltración local de corticoides, para el síndrome del túnel del carpo, en población trabajadora colombiana. Teniendo en cuenta que es una de las enfermedades de origen laboral más prevalentes en el país, se realizó un estudio de costo efectividad, desde la perspectiva del tercer pagador (Empresas Promotoras de Salud y Aseguradoras de Riesgos Laborales), en que se compararon alternativas de tratamiento quirúrgica y médica, para esta patología, para lo cual, se elaboró un árbol de decisión en el escenario clínico de un trabajador con un síndrome del túnel del carpo severo, para un horizonte temporal de un año, en el que se incluyeron los costos directos e indirectos asociados. Los resultados para el escenario y horizonte temporal propuesto en relación con esta patología es que ninguna de las alternativas resultó ser claramente dominante, dentro del plano de costo-efectividad. La razón de costo-efectividad incremental de la cirugía en comparación con la aplicación de corticoides fue de $\$ 1.974 .945,68$, por 0,6 casos de mejoría. En el análisis de sensibilidad de Montecarlo, la aplicación local de corticoides fue costo-efectiva en el $100 \%$ de los casos, de acuerdo con el umbral propuesto.
Palabras clave: Síndrome del túnel carpiano, evaluación de la tecnología biomédica, evaluación de costo-efectividad (DeCS - www.decs.bvs.br).

\section{SUMMARY}

Carpal tunnel syndrome (CTS) is the most common entrapment neuropathy and in turn, the one that most affects the working population whose treatment implies an economic burden to the health system. The aim of this research was to evaluate the cost-effectiveness of conventional surgical treatment and local infiltration of corticosteroids for carpal tunnel syndrome in the Colombian working population. Considering that it is one of the most prevalent occupational diseases, in the country from the perspective of the third payer (Health Promoting Companies and Occupational Risk Insurers). A cost-effectiveness study was carried out in which alternatives of surgical and medical treatment for this pathology were compared, through a decision tree was elaborated in the clinical scenario of a worker with a severe carpal tunnel syndrome for a time horizon of one year, in which the associated direct and indirect costs were included. The results from the scenario and time horizon proposed in relation to this pathology, none of the alternatives turned out to be dominant within the plane of cost-effectiveness. The incremental costeffectiveness ratio of the surgery compared to the application of corticosteroids was $1,974,945.68$ pesos for 0.6 cases of improvement. In the sensitivity analysis of Monte Carlo, the local application of corticosteroids was cost-effective in $100 \%$ of the cases according to the proposed threshold. 
Key-words (MESH): Cost-benefit analysis, carpal tunnel syndrome, technology assessment, biomedical.

\section{INTRODUCCIÓN}

El Síndrome de Túnel del Carpo (STC) es la neuropatía periférica por atrapamiento más frecuente, que afecta casi al $3 \%$ de la población general, aunque esta prevalencia puede aumentar al $15 \%$ en poblaciones trabajadoras, que están expuestas a factores de riesgo ocupacional y puede causar una significativa discapacidad relacionada con el trabajo (Kho et al. 2017). Hallazgos de diferentes estudios sitúan la prevalencia dependiendo del género, $9,2 \%$, en mujeres y $6 \%$, en hombres y en Colombia es la primera causa de morbilidad laboral (Ministerio de la Protección Social, 2007; Dominguez, 2012). Más allá de la exposición laboral hay factores de riesgo para el STC, como: género femenino, edad, diabetes, hipotiroidismo, obesidad, historia familiar de STC, menopausia, patologías concomitantes, como artritis reumatoide, baja talla, antecedente de tabaquismo y uso de anticonceptivos orales (Newington et al. 2015).

El STC, se caracteriza por un curso clínico no uniforme e impredecible (Ortiz-Corredor et al. 2008). Las medidas conservadoras que han sido consideradas para el tratamiento inicial de esta patología son los esteroides locales y sistémicos y antiinflamatorios no esteroideos. Los resultados de la liberación del nervio mediano en el túnel del carpo en términos de mejoría de los síntomas y puntajes funcionales se han justificado en personas mayores, pero los resultados quirúrgicos en jóvenes son menos predecibles. En una revisión sistemática que buscaba evaluar la eficacia del tratamiento quirúrgico para el STC versus el tratamiento no quirúrgico, en pacientes con esta condición de estadios leve a severo, se incluyó: ferulaje, modificación de actividades, antiinflamatorios no esteroideos, diuréticos e inyección de corticoides. La terapia quirúrgica comprendió las diferentes modalidades existentes, como la cirugía abierta y la endoscópica e, incluyendo, optativamente, la neurolisis. A los seis meses del tratamiento, 72 pacientes del grupo quirúrgico $(82,7 \%)$ reportaron mejorías frente a 57 (64\%), del grupo de férula (Verdugo et al. 2008).

El STC puede causar significativas disminuciones en la productividad de los trabajadores (Kobelt, 2012). Los rangos de retorno al trabajo pueden, en promedio, estar en 11 días para un trabajador que no realice una actividad predominantemente manual y que sea independiente y, en 72 días, para un trabajador en un proceso de reclamación (Chaise et al. 2004). Un estudio realizado en Colombia reporta una media de incapacidad temporal para un trabajador con esta enfermedad de 14 días (Palencia-Sánchez et al. 2013).
Los costos, usualmente asociados a esta patología, son: atención sanitaria, intervención quirúrgica y rehabilitación, que pueden ser elevados; por ejemplo, un estudio de costo-utilidad, comparando la cirugía endoscópica contra la cirugía abierta, para casos severos del túnel, encontró una razón de costo-utilidad incremental, de 124.311,32 dólares canadienses, por QALY, para la cirugía endoscópica, lo que la descarta por costos en relación a su efectividad (Thoma et al. 2006). Otro estudio de costo-utilidad en el que se comparó el tratamiento médico con terapia, férulas y aplicación local de corticoesteroides versus la cirugía abierta, en pacientes con STC, desde leve a moderado, halló una razón de costo-utilidad favorable, para el tratamiento quirúrgico (Pomerance et al. 2009).

Los estudios de costo-efectividad miden los desenlaces en unidades naturales, tales como casos de mejoría, prevenidos, muertes evitadas o eventos detectados y pueden ayudar a distribuir el cuidado de la salud y limitar los riesgos morales del aseguramiento, superando las limitaciones de la información; no obstante, los resultados de una evaluación económica no son extrapolables, ya que están sujetos a la diferencia de precios relativos entre países y al umbral de disponibilidad a pagar (Hunink et al. 2014). El objetivo de este estudio fue comparar el costo-efectividad entre el tratamiento quirúrgico convencional y la infiltración local con corticoides, para el STC, en la población trabajadora colombiana, desde la perspectiva del tercer pagador.

\section{MATERIALES Y MÉTODOS}

Para este estudio, se desarrolló una Evaluación Económica Completa de Tecnologías en Salud (EETS) del subtipo costo-efectividad. Se realizó una EETS, basada en datos secundarios, desde la perspectiva del tercer pagador (Aseguradora de Riesgos Laborales -ARL- y Empresas Promotoras de Salud -EPS-, del Sistema General de Seguridad Social en Colombia). Se tomó como población de referencia, a los trabajadores colombianos, independientemente si el origen de su patología era laboral o no y sin comorbilidad asociada al STC.

Las opciones terapéuticas incluidas en esta investigación son la liberación quirúrgica convencional en comparación con la infiltración local con corticoides. El escenario propuesto es un "STC severo" y, con este, se generó el modelo de costo-efectividad: en el modelo, se buscó que se compararan las alternativas terapéuticas descritas y sus desenlaces, en un horizonte temporal a un año, para así poder ser comparado frente a estudios similares realizados en otras latitudes (Korthals de Bos et al. 2006).

La identificación y la evaluación de las probabilidades, en cuanto a efectividad y seguridad de los tratamientos analiza- 
dos para el STC y así integrarlas al modelo, se realizaron por medio de búsquedas sistemáticas de publicaciones, especialmente, revisiones sistemáticas y metaanálisis (Verdugo et al. 2008; Shin \& MacDermind, 2011; Huisstede et al. 2010). Para la evaluación de la calidad de las revisiones sistémicas, se usó la herramienta AMSTAR, el acrónimo en inglés de "A Meaurement Tool to Assess systematic Reviews" (Shea et al. 2009). Para el diseño del modelo analítico, se elaboró un árbol de decisiones, debido a que el STC no tiene estadios de transición definidos y algunos pacientes, con esta patología, experimentan síntomas, mientras otros presentan períodos silentes con exacerbaciones de la sintomatología (Braun et al. 1989). Para tal fin, se revisaron las guías de atención vigente y se acudió al consenso informal de expertos (cirujanos de mano, ortopedistas o cirujanos plásticos, fisiatras, médicos laborales, fisioterapeutas y terapeutas ocupacionales expertos en calificación de pérdida de la capacidad laboral) (Ministerio de la Protección Social, 2007). Para el desarrollo del modelo propuesto, se tuvieron en cuenta los siguientes supuestos: exclusión de pacientes con STC con patologías asociadas a este, como hipotiroidismo, diabetes o artritis reumatoide; se consideró solamente la afectación de una extremidad por el STC; se asume que la no resolución o el retorno de los síntomas no es el resultado de la ausencia de diagnóstico de otra condición similar al STC; no se discriminó el género del trabajador afectado y los eventos adversos considerados fueron los que la literatura reportara como los más frecuentes. La secuencia para desarrollar el modelo analítico de decisión tuvo presente el algoritmo propuesto, para realizar evaluaciones económicas en salud (Hunink et al. 2014).

Para la identificación y la cuantificación de los eventos generadores de costos, de acuerdo con la perspectiva de la evaluación económica, se utilizaron los tarifarios del manual SOAT y de medicamentos, con los respectivos ajustes, relacionados con el aumento del Índice de Precios al Consumidor, para el año en que se realizó el estudio. Para efectos de esta investigación, la metodología del capital humano fue la escogida para el cálculo de estos costos indirectos (Drummond et al. 2015); las variables incluidas en cada una de las ramas del árbol de decisión, se especifican en la tabla 1 . En la figura 1, se observa el modelo diseñado para este estudio, que permite la comparación de corticoides locales frente a la cirugía convencional, en el caso trabajador que desempeña labores manuales, con un STC severo, que devenga un salario mínimo mensual vigente para el 2013 y los posibles desenlaces clínicos que pueden darse en esta situación, tales como mejoría clínica, falla del tratamiento, eventos adversos y recurrencia de la condición.

Para el desarrollo de esta investigación, se tomó como umbral de disponibilidad a pagar, la Unidad de Pago por Capi- tación (UPC), para el 2013, cuyo valor era \$595.969, aproximadamente, $\$ 600.000$. Este Umbral, escogido por el equipo interdisciplinario a cargo de este proyecto, permitió tener unos puntos de corte, que hicieran viable ejecutar los análisis de sensibilidad, con el objeto de profundizar en la comparación entre las dos alternativas consideradas, además, porque la UPC es el valor anual que se reconoce por cada uno de los afiliados al Sistema General de Seguridad Social en Salud (SGSS), para cubrir sus prestaciones en salud (Ministerio de Salud y Protección Social, 2012), por lo que se hizo el supuesto, que puede ser un proxy del umbral de disposición a pagar por el sistema de salud en Colombia y, por último, porque los umbrales de disposición a pagar, sugeridos por la OMS, son más idóneos, cuando se están realizando EETS, para enfermedades de alto costo, como el cáncer, especialmente, en estudios de costo-utilidad. Finalmente, se realiza un análisis de sensibilidad probabilístico, para evaluar la incertidumbre global del modelo (Drummond et al. 2015).

\section{RESULTADOS Y DISCUSIÓN}

A continuación, se describe los resultados obtenidos con el programa TreeAge Pro-2011®, con el cual, se corrió el modelo analítico de decisión, diseñado para el escenario a estudio, como es el de un trabajador que realiza una labor manual, diagnosticado con un STC severo y proyectando los desenlaces clínicos a un año, teniendo en consideración, que los modelos generados por este software son ampliamente aceptados en la comunidad científica y las agencias regulatorias, para la toma de decisiones en estudios de EETS (TreeAge Software INC, 2013).

En la figura 2, se observan los resultados del análisis de costo-efectividad, al correr el modelo diseñado para el escenario propuesto. Para este caso, la aplicación de corticoides, a nivel local, se ubica en el plano de una intervención menos efectiva y, a la vez, menos costosa, a diferencia de la cirugía, por lo que la toma de decisiones acerca de la opción a escoger, no es tan evidente y, por consiguiente, la alternativa a elegir, dependerá de su efectividad y que tenga una RCEI más baja, que la disponibilidad a pagar. En cuanto a la disponibilidad a pagar, la Organización Mundial de la Salud -OMS- propone que, si una tecnología tiene una RCEI de hasta un Producto Interno Bruto -PBI- per cápita por años de vida ajustados por discapacidad (AVAD), es muy costo-efectiva; si la misma está entre uno y dos PIB/AVAD evitado, es costo-efectiva y si es mayor a tres PIB/AVAD, no es costo-efectiva. En general, esto se suele usar también en forma equivalente, para estimar el umbral de costo por QALY o año de vida y se suele emplear en estudios de costo-utilidad (Pichón \& Augustovski, 2012). Para Colombia, según datos del Banco Mundial, su PIB, para el 2013, era de 8.030,58 lo que equivale al cambio, del aquel entonces, a \$15.507.049,9 (Banco Mundial, 2017). 
Tabla 1. Variables incluidas por rama del modelo analítico de decisión, para la EETS.

\begin{tabular}{|c|c|c|c|}
\hline Costos directos & $\begin{array}{l}\text { Límite Inferior Pesos COL. } \\
\text { Se calculó sobre el valor } \\
\text { tope de los tarifarios a } \\
2013 \text { menos el } 30 \%\end{array}$ & $\begin{array}{c}\text { Valor Base Pesos COL. Se } \\
\text { calculó sobre el valor tope } \\
\text { de los tarifarios a } 2011 \\
\text { menos el } 15 \%\end{array}$ & $\begin{array}{c}\text { Límite superior Valor } \\
\text { Tarifario } 2013 \text {. Se calculó } \\
\text { como valor máximo o valor } \\
\text { tope la tarifa plena de los } \\
\text { tarifarios a } 2013\end{array}$ \\
\hline $\begin{array}{l}\text { Costo tratamiento con Infil- } \\
\text { tración local corticoide }\end{array}$ & $184.691,13$ & $224.905,05$ & $282.490,97$ \\
\hline $\begin{array}{l}\text { Costo tratamiento con In- } \\
\text { filtración corticoide más } \\
\text { evento adverso }\end{array}$ & $196.913,13$ & $238.723,05$ & $297.778,97$ \\
\hline $\begin{array}{l}\text { Costo tratamiento quirúrgico } \\
\text { convencional }\end{array}$ & $651.851,20$ & $791.533,60$ & 931.216 \\
\hline $\begin{array}{l}\text { Costo tratamiento quirúrgico } \\
\text { convencional más evento } \\
\text { adverso }\end{array}$ & $1.077 .853,70$ & $1.308 .822,35$ & 1.539 .791 \\
\hline Costos indirectos & $\begin{array}{l}\text { Días de incapacidad } \\
\text { temporal. Mínimo (12) }\end{array}$ & $\begin{array}{c}\text { Días de incapacidad } \\
\text { temporal. Promedio } \\
\text { (14) }\end{array}$ & $\begin{array}{l}\text { Días de incapacidad } \\
\text { temporal. Máximo (144) }\end{array}$ \\
\hline $\begin{array}{l}\text { Costo del ausentismo a } \\
\text { causa del STC = Días de } \\
\text { Incapacidad temporal por } \\
\text { salario mínimo diario vigente } \\
2013\end{array}$ & 235.000 & 275.100 & 2.829 .600 \\
\hline $\begin{array}{c}\text { Desenlaces clínicos con- } \\
\text { siderados }\end{array}$ & Probabilidad de ocurrencia & \multicolumn{2}{|c|}{ Intervalo de confianza $95 \%$} \\
\hline $\begin{array}{l}\text { Mejoría con la cirugía de } \\
\text { liberación para el STC a los } \\
\text { doce meses }\end{array}$ & 0,77 & 0,66 & 0,85 \\
\hline $\begin{array}{l}\text { Mejoría con la inyección de } \\
\text { corticoides a los doce meses }\end{array}$ & 0,63 & 0,45 & 0,79 \\
\hline $\begin{array}{l}\text { Complicaciones quirúrgicas } \\
\text { de forma global }\end{array}$ & 0,02 & 0,002 & 0,07 \\
\hline $\begin{array}{l}\text { Complicaciones tras apli- } \\
\text { cación de corticoides locales } \\
\text { de forma global }\end{array}$ & 0,001 & 0,00001 & 0,0056 \\
\hline $\begin{array}{l}\text { Recurrencia del STC tras la } \\
\text { aplicación de corticoides }\end{array}$ & 0,5 & 0,23 & 0,63 \\
\hline $\begin{array}{l}\text { Recurrencia del STC tras la } \\
\text { cirugía }\end{array}$ & 0,057 & 0,016 & 0,112 \\
\hline
\end{tabular}

Fuente: Adaptado de Manual SOAT. 2013 (Presidencia de la República de Colombia, 1996); Verdugo et al. 2008; Shin \& MacDermind, 2011; Huisstede et al. 2010. 


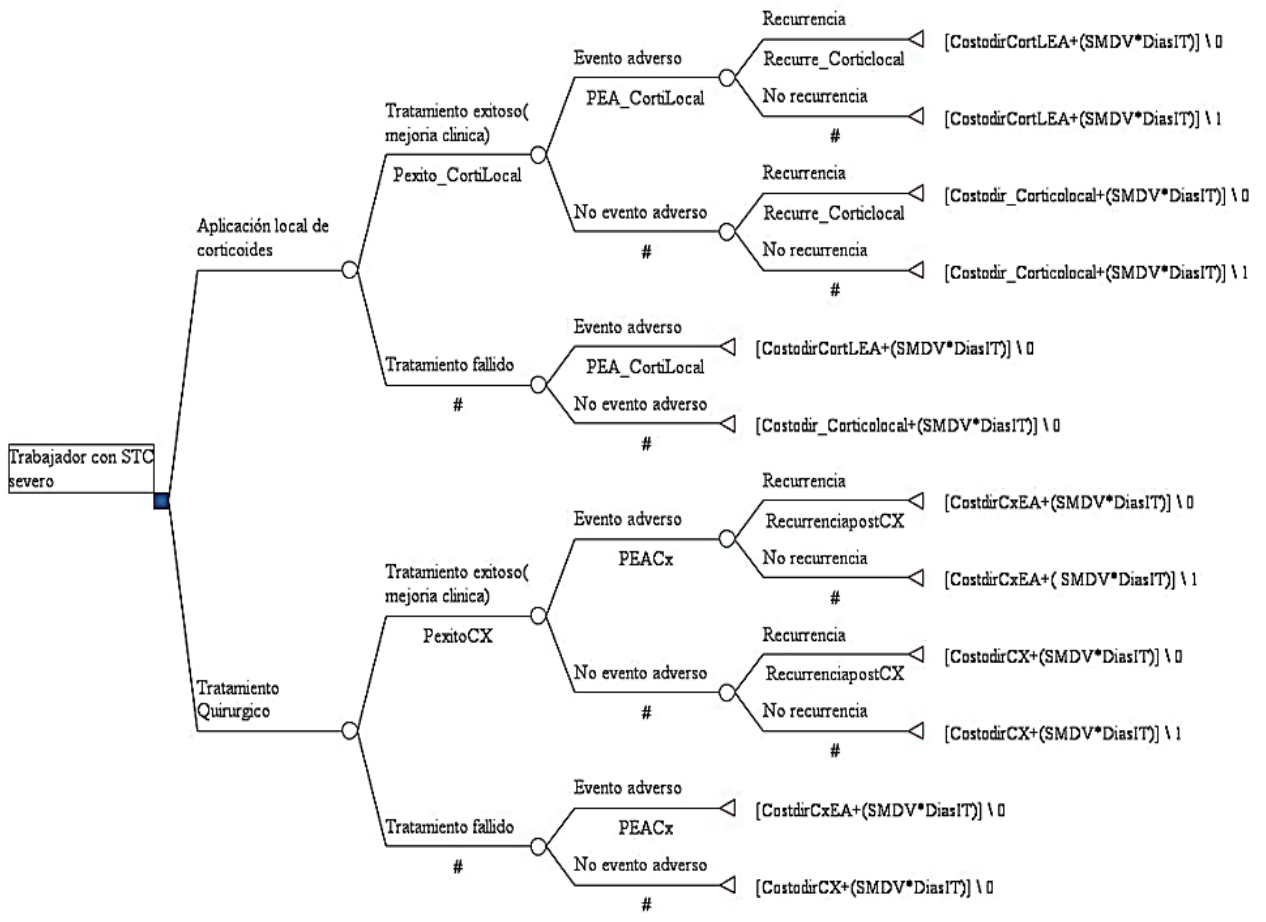

CostodirCortLEA: Costo directo aplicación corticoides-CostodirCX: costo directo de cirugía- DíasIT: Días Incapacidad Temporal promedio -PEACortiLocal: Probabilidad de Evento Adverso Corticoide Local-PEACX: Probabilidad de evento adverso cirugía-Pexito cortilocal: probabilidad de éxito del tratamiento con corticoides-Pexito CX: probabilidad de éxito con la cirugía convencional-Recurre_Corticlocal: probabilidad de recurrencia tras aplicación de corticoide local-RecurrenciapostCX: probabilidad de recurrencia tras liberación quirúrgica-SMVD: Salario Minimo Vigente Diario para el 2013

Figura 1. Modelo analítico de decisión, considerando a un trabajador con STC severo y desenlaces a 12 meses.

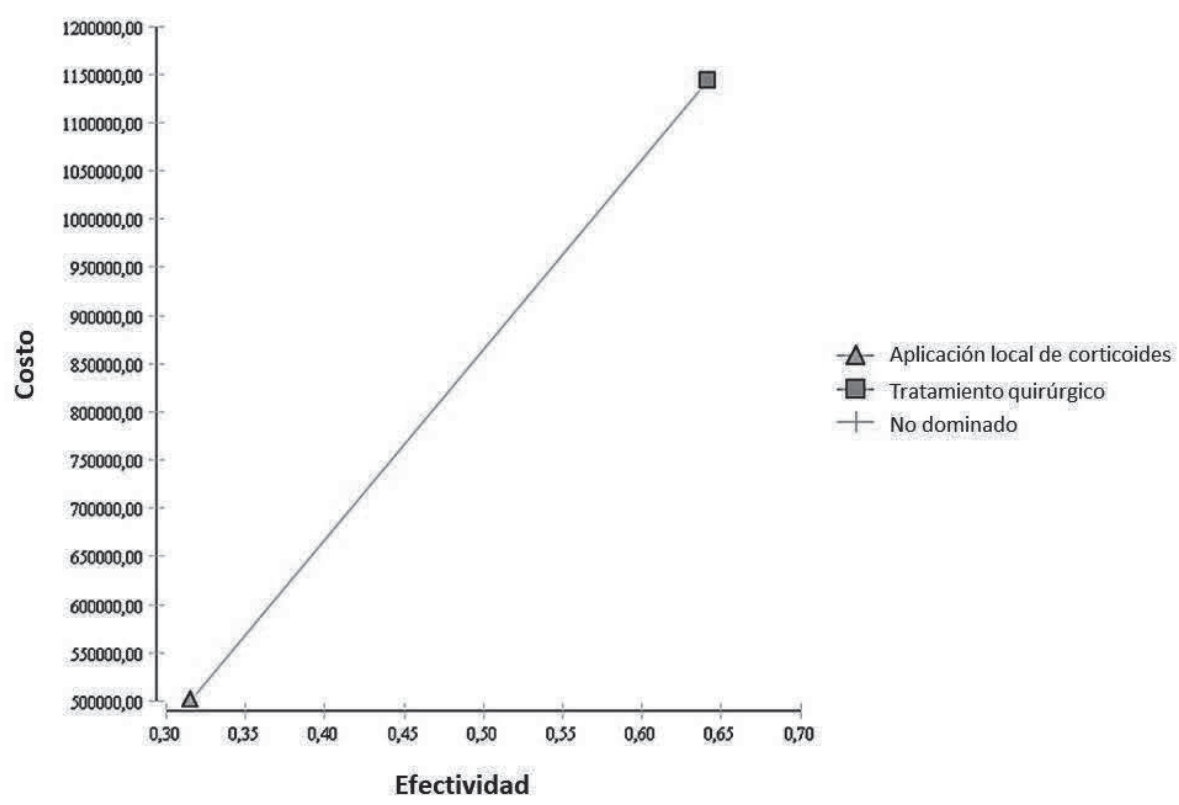

Figura 2. Representación de los resultados en el plano de costo-efectividad, al correr el análisis del caso a estudio. 
En el escenario propuesto, con un horizonte de resultados a un año, para el caso de un trabajador con STC severo, en quien se compara una opción médica de tratamiento (aplicación de corticoides), contra la opción quirúrgica convencional, la razón de costo-efectividad incremental (RCEI o ICER, por sus siglas en idioma inglés) de la cirugía, en comparación con la aplicación de corticoides locales, es de $\$ 1.974 .945,68$ colombianos, por 0,64 caso de mejoría. En la tabla 2, se presentan los resultados de costo-efectividad, para dicho escenario.
Análisis de sensibilidad a dos vías en el escenario analizado: Al realizar el análisis a dos vías, con un umbral de disposición a pagar entre una y dos UPC, el resultado que mostró ser más costo-efectivo es la aplicación local de corticoides (Figura 3). En esta, se muestra en el eje X, los valores de eficacia del tratamiento, con la aplicación del corticoide local y, en el eje Y, los valores de eficacia del tratamiento, con la aplicación de la cirugía convencional. Las áreas con distinto color representan aquellas combinaciones de valores, en

Tabla 2. Resultados de costo-efectividad en el caso base, en el escenario analizado.

\begin{tabular}{|c|c|c|c|c|c|c|c|}
\hline Estrategia & Dominio & $\begin{array}{c}\text { Efectividad } \\
\text { (caso de } \\
\text { mejoría) }\end{array}$ & $\begin{array}{c}\text { Efectividad } \\
\text { incremental }\end{array}$ & Costo & $\begin{array}{c}\text { Costo } \\
\text { Incremental }\end{array}$ & $\begin{array}{c}\text { Razón CE } \\
\text { incremental }\end{array}$ & $\begin{array}{c}\text { Razón CE } \\
\text { promedio }\end{array}$ \\
\hline $\begin{array}{c}\text { Aplicación } \\
\text { local de } \\
\text { corticoides }\end{array}$ & $\begin{array}{c}\text { No } \\
\text { dominado }\end{array}$ & 0,31 & 0 & $500,018,818$ & 0 & 0 & $1,587,361,33$ \\
\hline $\begin{array}{c}\text { Tratamiento } \\
\text { quirúrgico } \\
\text { convencional }\end{array}$ & $\begin{array}{c}\text { No } \\
\text { dominado }\end{array}$ & 0,64 & 0,32619 & $1,144,226,35$ & $644,207,532$ & $1,974,945,68$ & $1,784,535,55$ \\
\hline
\end{tabular}

Fuente: Cálculo de los autores.

las cuales, una u otra estrategia son costo-efectivas. En este caso, se observa que el área de color rojo es donde es más costo-efectiva la opción quirúrgica y, el área azul, donde es más costo-efectiva la aplicación local de corticoides. Se ob- serva que entre más alta sea la eficacia de la aplicación de corticoides, independientemente del valor de la eficacia de la cirugía, será la alternativa más costo efectiva.

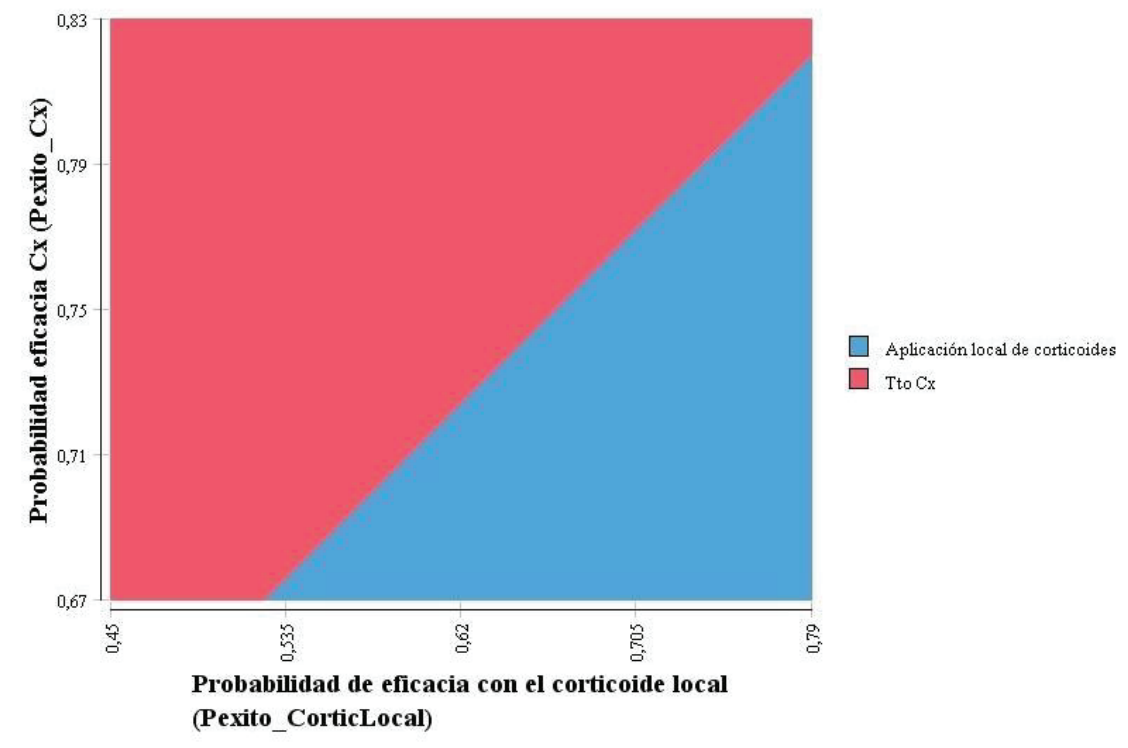

Figura 3. Resultado gráfico del análisis de sensibilidad a dos vías, en el escenario a estudio. 
Análisis de sensibilidad probabilístico para el escenario analizado: En el análisis probabilístico (simulación de Montecarlo), con un valor de disponibilidad a pagar de una UPC, el resultado que se muestra en la curva de aceptabilidad (Figura 4), indica que la opción a escoger, para el escenario propuesto por esta investigación, es la aplicación local de corticoides, es del $100 \%$.
En la actualidad, podemos identificar que, a pesar de las investigaciones realizadas, siguen existiendo controversias sobre cuáles son las mejores intervenciones respecto al tratamiento más costo-efectivo en el manejo del STC y, por ende, cuál debe ser la provisión de servicios sanitarios, relacionados con esta enfermedad (Prime et al. 2010). Las consecuencias económicas relacionadas con esta patología

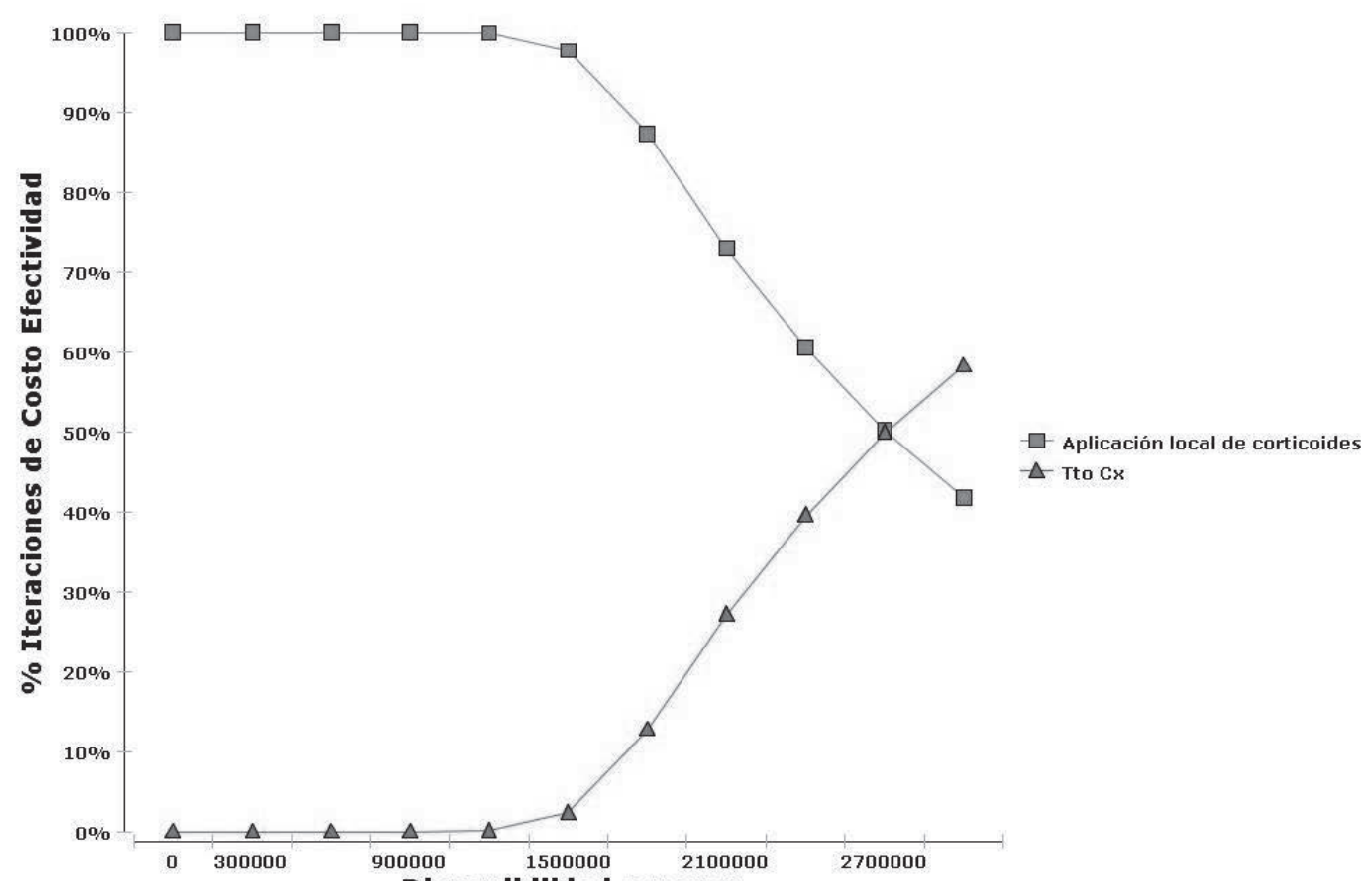

Figura 4. Curva de aceptabilidad estrategias para el escenario propuesto para análisis, en la presente investigación.

incluyen las implicaciones directas del financiamiento de los tratamientos y la carga indirecta del ausentismo (Palencia-Sánchez et al. 2013). En Holanda, se realizó una evaluación económica dentro de un ensayo clínico aleatorizado controlado, comparando la cirugía con el uso de férulas en el tratamiento del STC, observando, que la razón de costo-efectividad favoreció al tratamiento quirúrgico (Korthals de Bos et al. 2006).

En este estudio de costo-efectividad, se tomó la perspectiva del tercer pagador (perspectiva en la que se puede estimar, de forma más precisa, los costos), ya que se genera una mayor validez de los resultados, cuando estos costos son incorporados al modelo (Drummond et al. 2015). Para la realización de este trabajo, se determinaron los costos del tratamiento, a partir de varias fuentes: Ministerio de la Protección Social, ARL y EPS, las cuales, se contrastaron con el levantamiento de costos que se realizó, de acuerdo con el tarifario SOAT 2013 (Presidencia de la República de Colom- bia, 1996), con el propósito de disminuir los sesgos y que el estudio tuviera una mayor validez externa; sin embargo, no se desconoce el hecho que abordar una perspectiva social pudiera aportar más elementos para la toma de decisiones, tal como lo reconocen diversos autores, en el campo de la economía de la salud. La literatura muestra que las intervenciones terapéuticas para el manejo del STC pueden estar relacionadas con eventos adversos, que pueden influir, de modo importante, en la calidad de vida y en los costos, para los sistemas sanitarios, por lo que es importante tenerlos en cuenta, al momento de diseñar un modelo analítico de decisión, en una EETS, criterio que se incluyó en el modelo (Verdugo et al. 2008).

Si bien es cierto que el STC, según algunos autores, no se considera como una enfermedad de alto costo en Colombia (Gallardo Solarte et al. 2016), esta patología conlleva una carga importante de enfermedad, porque afecta población económicamente activa, lo que puede disminuir su capaci- 
dad laboral, incidiendo no solamente en el menoscabo de su estado de salud sino también en la capacidad productiva. Para el caso analizado, los umbrales de disposición a pagar, propuestos por la OMS, se pueden considerar de poca utilidad para la toma de decisiones, porque el STC es una enfermedad que no se considera de alto costo; sin embargo, los datos epidemiológicos que muestran la carga de la enfermedad y costos asociados a esta, en Colombia son importantes, lo cual, impulso al grupo interdisciplinario, para este caso, proponer como umbral de disposición a pagar a la UPC, como una innovación metodológica de este estudio, de tal forma, que permitiera realizar los análisis de sensibilidad, especialmente, el de tipo Montecarlo y, de esta forma, facilitar la toma de decisiones, en la escogencia del tratamiento (datos si publicar).

En el presente estudio, se escogió el salario mínimo diario legal vigente, para el 2013, como referencia para el cálculo de los costos indirectos, porque esta patología es más frecuente en trabajadores que hacen labores manuales que, por lo usual, son los que perciben una menor retribución económica (Violante et al. 2016). Esto constituye, otra diferencia en el desarrollo metodológico de la presente EETS, con otros estudios previos, tales como el de Pomerance et al. 2009), que compara intervenciones quirúrgicas como no quirúrgicas o la realizada por un grupo de investigación holandés, comparando el uso de la férula con la cirugía (Korthals de Bos et al. 2006), las que no consideran estos costos indirectos en detalle.

Como conclusión del presente análisis de costo-efectividad encontramos que, al evaluar el costo-efectividad de las opciones terapéuticas propuestas en esta investigación para el STC, tanto la aplicación local de corticoides como la cirugía, mostraron ser intervenciones muy costo-efectivas, de acuerdo con los criterios de la Organización Mundial de la Salud.

Cuando se toma como umbral de disposición a pagar la UPC, la opción más costo-efectiva es la aplicación de corticoides locales, una decisión apoyada por los resultados de los análisis de sensibilidad, a dos vías y por la simulación de Montecarlo. Lo notorio de este resultado es que es apoyado por estudios realizados posteriormente, como el adelantado en Turquía, comparando las mismas opciones terapéuticas (Gurcay et al. 2017). En el caso de ampliar el umbral de disposición a pagar a tres veces la UPC, la opción a escoger sería la cirugía convencional de liberación del STC.

Una limitación en particular de la presente EETS, se debe a que como el STC es una enfermedad que se ha relacionado con la exposición laboral, en la legislaciones de los países occidentales, incluyendo a Colombia, se han observado normas legales de protección y de compensación a los trabajadores, quienes pueden tener influencia sobre los desenlaces del tra- tamiento del STC, por cuanto el trabajador puede no expresar fielmente los resultados de la intervención a la que se somete, generando un sesgo de información (Kho et al. 2017).

Conflictos de intereses: El manuscrito fue preparado y revisado con la participación de todos los autores, quienes declaramos que no existe ningún conflicto de intereses que ponga en riesgo la validez de los resultados presentados. Financiación: Para el desarrollo de esta investigación no se recibió financiación.

\section{Consideraciones éticas}

Esta investigación, se basó en datos secundarios y no requirió la participación de personas para su desarrollo, en coherencia con la resolución 8430 de 1993, del Ministerio de Salud de Colombia, se puede clasificar como sin riesgo. Además, tuvo el aval del Comité de Ética de la Facultad de Medicina de la Universidad Nacional de Colombia.

\section{BIBLIOGRAFÍA}

1. BANCO MUNDIAL. 1 de Enero de 2017. PIB per cápita (US\$ a precios actuales) Disponible desde Internet en: https://datos.bancomundial.org/indicador/ NY.GDP.PCAP.CD?locations $=\mathrm{CO}$ (con acceso el 11/12/17).

2. BRAUN, R.M.; DAVIDSON, K.; DOEHR, S. 1989. Provocative testing in the diagnosis of dynamic carpal tunnel syndrome. J. Hand Surgery. (Am.) 14:195-197.

3. CHAISE, F.; BELLEMERE, P.; FRIL, J.; GAISNE, E.; POIRIER, P.; MENADI, A. 2004. Return-to-work interval and surgery for carpal tunnel syndrome. Results of a prospective series of 233 patients. J. Hand Surg [Br]. 29:568-70.

4. DOMINGUEZ, J. 2012. Para que las manos no duelan. Disponible desde Internet en: http://www.sura.com/ blogs/calidad-de-vida/tunel-carpiano.aspx (con acceso el 05/09/16).

5. DRUMMOND, M.F.; SCULPHER, M.J.; CLAXTON, K.; STODDART, G.L.; TORRANCE, G.W. 2015. Methods for the economic evaluation of health care programmes ( $4^{\text {th }}$ edition). Oxford University Press. 464p.

6. GALLARDO SOLARTE, K.; BENAVIDES ACOSTA, F.P.; ROSALES JIMÉNEZ, R. 2016. Costos de la enfermedad crónica no transmisible: la realidad colombiana. Rev. Ciencias de la Salud. 14(1):103-114.

7. GURCAY, A.G.; KARAAHMET, O.Z.; GURCAN, O.; KAZANCI, A.; KARSLI, P.B.; UMAY, E.K.; CAKCI, A. 
2017. Comparison of short-term clinical and electrophysiological outcomes of local steroid injection and surgical decompression in the treatment of carpal tunnel syndrome. Turk Neurosurg. 27(3):447-452.

8. HUISSTEDE , B.M.; HOOGVLIET, P.; RANDSDORP, M.S.; GLERUM, S.; VAN MIDDELKOOP, M.; KOES, B.W. 2010. Carpal tunnel syndrome. Part I: effectiveness of nonsurgical treatmentsa systematic review. Arch Phys Med Rehabil. 91(7):981-1004.

9. HUNINK, M.M.; WEINSTEIN, M.C.; WITTENBERG, E.; DRUMMOND, M.F.; PLISKIN, J.S.; WONG, J.B.; GLASZIOU, P.P. 2014. Decision making in health and medicine: integrating evidence and values $\left(2^{\text {nd }}\right.$ edition). Cambridge University Press. 424p.

10. KHO, J.Y.; GASPAR, M.P.; KANE, P.M.; JACOBY, S.M.; SHIN, E.K. 2017. Prognostic Variables for Patient Return-to-Work Interval Following Carpal Tunnel Release in a Workers' Compensation Population. HAND. 12(3):246-251.

11. KOBELT, G. 2012. Economic studies in rheumatology: data perspectives challenges. Rheumatology. 51:208-209.

12. KORTHALS DE BOS, I.; GERRRITSEN, A.; VAN TULDER, M.; RUTTEN VAN MOLKEN, M.; ADER, H.; DE VET, H. 2006. Surgery is more cost effective than splinting for carpal tunnel syndrome in the Netherlands:reult of an economic evaluation alongside a randomized controlled trial. BMC Musculoskeletal disorders. 7(86):1-9.

13. MINISTERIO DE LA PROTECCIÓN SOCIAL. 2007. Informe de enfermedad profesional en Colombia 2003 - 2005. Dirección General de Riesgos Profesionales, Bogotá.

14. MINISTERIO DE LA PROTECCIÓN SOCIAL. 2007. Guia de atención integral basada en la evidencia para desórdenes (DME) relacionados con movimientos repetitivos de miembros superiores. Guía, Bogotá.

15. MINISTERIO DE SALUD Y PROTECCIÓN SOCIAL. 2012. Resolución 4480 de 2012. Por la cual se fija el valor de la UPC del POS de los Regímenes Contributivos y Subsidiado para el año 2013 y se dictan otra disposiciones.

16. NEWINGTON, L.; HARRIS, E.C.; WALKER-BONE, K. 2015. Carpal tunnel syndrome and work. Best Practice \& Research Clinical Rheumatology. 29(3):440-453.
17. ORTIZ-CORREDOR, F.; ENRÍQUEZ, F.; DÍAZ-RUÍZ, J.; CALAMBAS, N. 2008. Natural evolution of carpal tunnel syndrome in untreated patients. Clinical Neurophysiology. 119:1373-1378.

18. PALENCIA-SÁNCHEZ, F.; GARCÍA-VEGA, O.A.; RIAÑO-CASALLAS, M.I. 2013. Carga de la Enfermedad Atribuible al Síndrome de Túnel del Carpo en la Población Trabajadora Colombiana: Una Aproximación a los Costos Indirectos de una Enfermedad. Value in Health Regional Issues. 2(3):381-386.

19. PICHÓN, A.; AUGUSTOVSKI, F. 2012. Introducción a las Evaluaciones Económicas en ETS. Curso de Evalauciones Económicas, Instituto de Efectividad Clínica y Sanitaria.

20. POMERANCE, J.; ZURAKOWSKI, D.; FINE, I. 2009. The Cost-Effectiveness of Nonsurgical Versus Surgical Treatment for Carpal Tunnel Syndrome. J. Hand Surg. 34:1193-1200.

21. PRESIDENCIA DE LA REPÚBLICA DE COLOMBIA. 1996. Decreto 2423 del 31 de diciembre de 1996. Disponible desde Internet en: http://www.hrd.gov.co/ documentos/facturacion/MANUAL\%20DE\%20FACTURACION\%202013/MANUAL\%20SOAT\%202013. pdf. (con acceso el 02/06/13).

22. PRIME, M.; PALMER, J.; KHAN, W.; GODDARD, N. 2010. Is there light at the end of the tunnel? Controversies in the Diagnosis and Management of Carpal Tunnel Syndrome. Hand. 5(4):354-360.

23. SHEA, B.; HAMEL, C.; WELLS, G.; BOUTER, L.; GRIMSHAW, J.; KRISTJANSSON, E. 2009. AMSTAR is a reliable and valid measurement tool to asses the methodologicla quality of systematic review. J. Clinical Epidemiology. 62(3):1013-1020.

24. SHIN, Q.; MACDERMIND, C.J. 2011. Is surgical intervention more effective than non-surgical treatment for carpal tunnel syndrome? A systematic review. J. Orthop. Surg. Res. 6(17):1-9.

25. THOMA, A.; WONG, V.; SPRAGUE, S.; DUKU, E. 2006. A cost-utility analysis of open and endoscopic carpal. Can. J. Plas.t Surg. 14(1):15-20.

26. TREEAGE SOFTWARE INC. 2013. Disponible desde Internet en: http://www.treeage.com/discover/ (con acceso el 09/06/13). 
27. VERDUGO, R.; SALINAS, R.; CASTILLO, J.; CEA, J. 2008. Surgical versus non-surgical treatment for carpal tunnel syndrome. Cochrane Database Syst Rev. 2008, Issue 4. Art. No.: CD001552.
28. VIOLANTE, F.S.; FARIOLI, A.; GRAZIOSI, F.; MARINELLI, F.; CURTI, S.; ARMSTRONG, T.J.; BONFIGLIOLI, R. 2016. Carpal tunnel syndrome and manual work: the OCTOPUS cohort, results of a ten-year longitudinal study. Scandinavian J. Work, Environment \& Health, 42(4):280-290.

Recibido: Diciembre 13 de 2017

Aceptado: Mayo 15 de 2018

\section{Cómo citar:}

Palencia-Sánchez, F.; García-Vega, O.; Riaño-Casallas, M. 2018. Costo-efectividad del tratamiento quirúrgico vs corticoides locales en el síndrome del túnel del carpo. Rev.U.D.C.A ct. \& Div. Cient.21(1): 5-14. 